\title{
Nonlinear Nonaxisymmetric Dynamos for Active Stars
}

\author{
David Moss \\ Mathematics Department, The University, Manchester M13 9PL, \\ United Kingdom
}

\begin{abstract}
Recent observations seem to have detected large scale nonaxisymmetric structures on active giant stars. These structures are plausibly associated with underlying, dynamo generated, nonaxisymmetric magnetic fields. Such developments have motivated the development of a computer code to solve the nonlinear mean field dynamo equation in spherical geometry with no imposed geometrical symmetries. The nonlinearity is a simple $\alpha$-quenching. The nature of the stable solutions found depends quite sensitively on the assumed spatial distribution of both $\alpha$-effect and differential rotation, and also on the degree of supercriticality of the dynamo. Such a dynamo model with stable, purely nonaxisymmetric solutions is described in this paper.
\end{abstract}

\section{Introduction}

The first aim of astrophysical dynamo theory has been to understand the solar magnetic cycle. Observations suggest that the mean solar field is approximately axisymmetric. Plausible kinematic models, in which large and small scale velocity fields are prescribed in an ad hoc but hopefully physically reasonable manner, can be constructed, but there is as yet no successful dynamically consistent model. These considerations have largely restricted the study of stellar dynamos to axisymmetric models, which does also allow considerable mathematical simplification.

Recently indicators of markedly non-axisymmetric surface structure have been detected in a few late type "active giant" (FK Comae) stars. Piskunov et al. (1990) have used surface imaging techniques to produce maps showing large-scale nonaxisymmetric surface temperature inhomogeneities on HD 32918. Jetsu et al. (1990) have found a 2.8 year variation in the amplitude of the photometric rotation modulation, superimposed on a 9 year cycle in the mean brightness. Phase seems to have been preserved for more than 13 years. Persistent active longitudes (a "flipflop" phenomenon) was found in FK Comae (Jetsu et al., in these Proceedings).

RS CVn stars, which are often in a state of spin-orbit synchronization, sometimes appear to have persistent spots at fixed longitudes (e.g. Zeilik, these Proceedings; and Hackman et al., these Proceedings). Assuming an analogy between 
the origin of sunspots and starspots, such observations can be interpreted as evidence for the presence of large-scale nonaxisymmetric stellar magnetic fields in these stars with deep convective envelopes.

It is known that in spherical dynamo models axisymmetric modes are often more easily excited than nonaxisymmetric. However Brandenburg et al. (1989) have demonstrated, for a strictly axisymmetric model, that a knowledge of linear growth rates is of very limited usefulness in determining the nonlinear stability of a system at supercritical dynamo number, and it can be anticipated that a similar situation exists when the restriction to axisymmetric fields is removed. By analogy, it may then be possible to find stable solutions consisting wholly or partly of nonaxisymmetric components.

\section{The Model}

The standard mean-field dynamo equation in dimensionless form

$$
\frac{\partial B}{\partial t}=\nabla \times(\boldsymbol{u} \times \boldsymbol{B}+\alpha \boldsymbol{B})-\nabla \times(\eta \nabla \times B)
$$

is solved in the spherical volume $0 \leq x \leq 1 . \tau$ is in units of the global diffusion time, $R^{2} / \eta, \alpha=\bar{\alpha} \tilde{\alpha}, \Omega=\bar{\Omega} \tilde{\Omega}, x=r / R$, where $R$ is the radius of the computational volume, $\eta$ is the uniform resistivity and $\bar{\alpha}$ and $\bar{\Omega}$ are typical values of $\alpha$ and $\Omega$. The dynamo parameters are given by

$$
C_{\alpha}=R \bar{\alpha} / \eta, \quad C_{\omega}=R^{2} \bar{\Omega} / \eta
$$

For the models described here

$$
\tilde{\alpha}=f(x) \cos \theta /\left[1+B^{2}(x, \theta, \lambda)\right],
$$

$x, \theta, \lambda$ being spherical polar coordinates, and

$$
\begin{gathered}
f(x)= \begin{cases}\frac{15}{16} \frac{1}{d_{\alpha}}\left(1-\xi_{\alpha}^{2}\right)^{2}, & \left|\xi_{\alpha}\right| \leq 1, \\
0 & \left|\xi_{\alpha}\right|>1,\end{cases} \\
\tilde{\Omega}(x)=\left\{\begin{array}{l}
-1, \text { for } \xi_{\Omega} \leq-1, \\
-\frac{1}{2}\left(1-\frac{3}{2} \xi_{\Omega}+\frac{1}{2} \xi_{\Omega}^{3}\right), \text { for }\left|\xi_{\Omega}\right| \leq 1, \\
0, \text { for } \xi_{\Omega} \geq 1,
\end{array}\right.
\end{gathered}
$$

where $\xi_{\alpha}=x-x_{\alpha} / d_{\alpha}$ and $\xi_{\Omega}=x-x_{\Omega} / d_{\Omega}$. More details are given in Moss et al. (1991). 


\section{Results}

The magnetic field can be split into parts that are symmetric (S) or antisymmetric (A) with respect to the rotational equator, and can be Fourier analysed into components proportional to $\exp (i m \lambda), m=0,1,2, \ldots$. (cf Stix, 1971). The gross field properties can be described by the parity parameter

$$
P=\left[E^{(\mathrm{S})}-E^{(\mathrm{A})}\right] /\left[E^{(\mathrm{S})}+E^{(\mathrm{A})}\right]
$$

and the symmetry parameter

$$
M=1-E_{0} / E
$$

where $E(S), E(A), E_{0}$ and $E$ are respectively the energies in the symmetric, antisymmetric, axisymmetric and total fields. For example, $P=+1 /-1$ denotes fields of pure even/odd parity and $M=0$ corresponds to a completely axisymmetric field.

Computations were performed for parameters $x_{\omega}=0.55, x_{\alpha}=0.75, d_{\omega}=0.25$, $d_{\alpha}=0.25, C_{\omega}=-150 . C_{\alpha}$ values for the excitation of the lowest axisymmetric and nonaxisymmetric modes are given in Table 1 . Supercritical values of $C_{\alpha}$ were taken, $5 \leq C_{\alpha} \leq 30$. Computations were started from a field of arbitrary structure, consisting of a mixture of axisymmetric and nonaxisymmetric parts. In each case the field evolved to a purely nonaxisymmetric S-type configuration, consisting of $m=1,3 \ldots$ components. Fig. 1 shows the evolution of $E_{0}, E_{N}=E-E_{0}, P$ and $M$ with time, and contours of constant surface field strength for a typical model are shown in Fig. 2. The solutions are steady in the sense that the total energy is constant, but the field structure rotates.

Table 1. Approximate critical values of $C_{\alpha}$ for excitation of linear modes and corresponding frequencies.

\begin{tabular}{|c|c|c|}
\hline Mode & $C_{\alpha, \text { crit }}$ & Frequency \\
\hline A0 & 4.48 & 18.4 \\
S0 & 4.13 & 13.2 \\
A1 & 3.98 & 0.57 \\
S1 & 3.94 & 0.68 \\
\hline
\end{tabular}



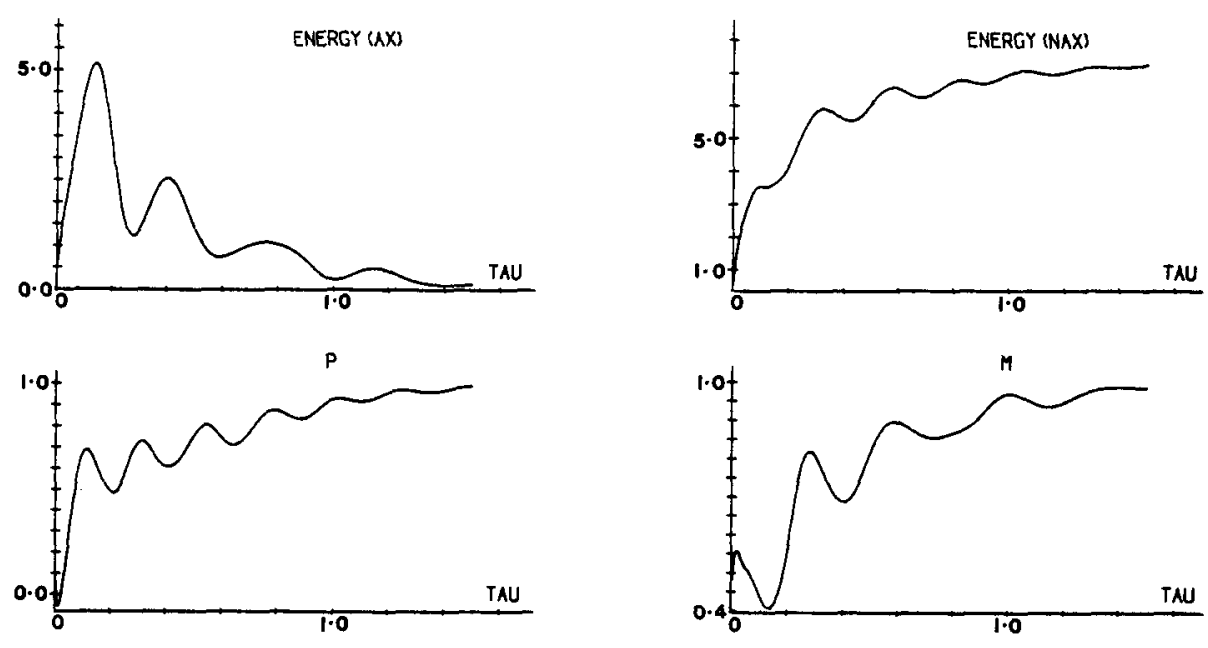

Fig. 1. Evolution with time of the energy in the axisymmetric and nonaxisymmetric parts of the field and aof the parameters $P$ and $M$ for model with $C_{\alpha}=30$.

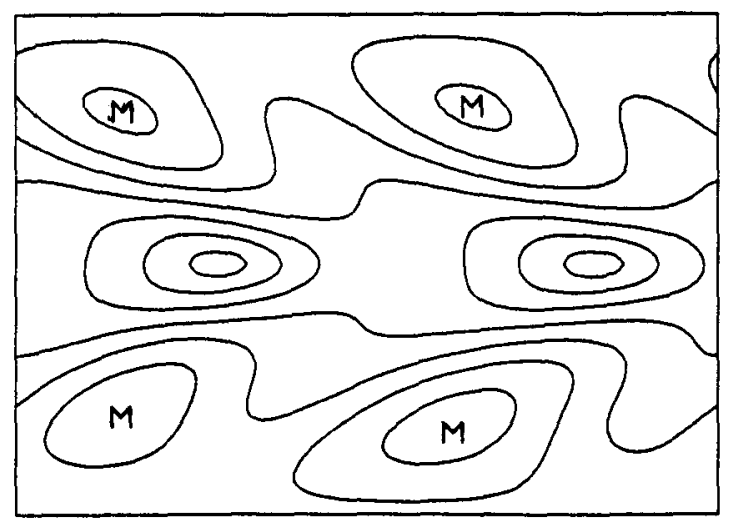

Fig. 2. Contours of equal surface field strength for the model with $C_{\alpha}=30$. Longitude runs horizontally and the top and bottom borders correspond to the north and south poles respectively. $M$ denotes regions where the field strength is highest. Slight equatorial asymmetries are because the field has not quite attained its final pure parity state.

\section{Discussion}

In a real stellar dynamo the nonlinear limitation of the field at finite amplitude may be caused by any (or all) of a number of mechanisms. Buoyancy, the effects of the Lorentz force on the small scale motions or on the differential rotation or 
meridional circulation are all possibilities. This model, with its very idealized $\alpha$ quenching mechanism, demonstrates the possibility of the existence of persistent nonaxisymmetric mean-field dynamo solutions. The existence of stable nonaxisymmetric solutions is very sensitive to the assumed source profiles, and also to the value of $C_{\omega}$. For example, in a series of experiments in which $x_{\alpha}, d_{\alpha}, d_{\omega}$ were kept fixed, for $x_{\omega}=0.40,0.65$ and 0.70 no stable nonaxisymmetric solutions were found, whereas for $x_{\omega}=0.50$ and 0.75 there are several nonaxisymmetric stable solutions but their properties can be somewhat different to those of the solutions described here with $x_{\omega}=0.55$ - see Moss et al. (1991) for details. Whether or not suitable $\alpha$ and $\Omega$ profiles can coexist as part of a dynamically self-consistent solution is a much deeper and more difficult question that is not tackled here.

It is known that strong differential rotation suppresses nonaxisymmetric modes (which is why the magnitude of $C_{\omega}$ in these models has been taken to be quite small. The nature of the differential rotation in the interior of the "active giants" is quite uncertain. However in the close, tidally interacting components of RS CVn systems it is probable that the differential rotation is relatively small. Very speculatively, this might favour the excitation of nonaxisymmetric dynamo fields and so explain the predominance of nonaxisymmetric structure ("active longitudes") in these stars.

Acknowledgement. I am grateful to Axel Brandenburg for performing linear calculations.

\section{References}

Brandenburg, A., Krause, F., Meinel, R., Moss, D., Tuominen, I.: 1989, Astron. Astrophys. 213, 411

Jetsu, L., Huovelin, J., Tuominen, I., Vilhu, O., Boppp., B.W., Piirola, V.: 1990, Astron. Astrophys. 236, 423

Moss, D., Tuominen, I., Brandenburg, A.: 1991, Astron. Astrophys., in press

Piskunov, N.E., Tuominen, I., Vilhu, O.: 1990, Astron. Astrophys. 230, 363

Stix, M.: 1971, Astron. Astrophys. 13, 203 